\title{
Barriers to Health Behavior Change in People with Type 2 Diabetes: Survey Study
}

\author{
Oumoukelthoum Mohamdy ${ }^{1,2^{*}}$
}

${ }^{1}$ Nursing Unit Manager, Nursing Department, Ministry of Health and Prevention, United Arab Emirates

${ }^{2}$ College of Health Solutions, Arizona State University, USA

*Corresponding author: Mrs. Oumoukelthoum Mohamdy, Nursing Unit Manager, Nursing Department, Ministry of Health and Prevention, Postal Code: 00971, United Arab Emirates

\begin{abstract}
Background: Diabetes mellitus DM (type 2) is one of the major chronic diseases that creates burden at the population level. A prevalence of 422 million patients with diabetes worldwide is detected [1]. UAE population has an increased prevalence of DM; According to International Diabetes Federation (IDF) $17.3 \%$ of the UAE population between the ages of 20 and 79 have type 2 diabetes in 2017 [2]. UAE has been suffering with the health problems that result from obesity and sedentary lifestyle. The purpose of this study is to understand barriers to health behavior among community patients with diabetes mellitus type 2 .
\end{abstract}

Methods: This is a qualitative survey study of 123 adult individuals with type 2 diabetes who are above 18-years-old, who participated in a survey that explore their perception about barriers to health behavior change. The survey was distributed online and in 6 health care centers under ministry of health and prevention in the United Arab Emirates.

Results: This study finds that the top barrier for behavior change is difficulty to follow healthy schedule (19.4\%), workplace condition and timing (14.3\%), and laziness (13\%). The least barrier was lack of knowledge about healthier behaviors $(5.3 \%)$. The best way to motivate behavior change was doctor's advice during regular visit $(26.3 \%)$, joining support group (18.4\%), and receiving electronic tips and reminders on smartphones (15\%).

Conclusion: The outcomes of this study can aid directly in community programs design and implementation, health education campaigns, and health policies. The findings of this study help in understanding real barriers to initiating and sustaining positive health behavior.

\section{Introduction}

Changing health behavior is an important step in disease prevention and management. Lifestyle-associated diseases are increasing because they are related to individuals' behavior and the difficulty to influence them. In type 2 diabetes, American Diabetes Association (ADA) guidelines recommend lifestyle modifications such as diet and exercise, as a foundation for DM type 2 management [3]. Teaching and educating diabetic patients about the importance of behavior change is crucial. In order to successfully change individuals' health behavior to healthier one, healthcare providers need to understand the concept of health behavior change and barriers to change. The concept of behavioral influence on the physical health and the appearance of diseases is not a new concept. It has been discussed and implemented long time ago. The term behavioral medicine had been first defined on 1977 on Yale conference [4]. The term was formally defined and stressed the important of behavioral-science knowledge application in diagnosis, prevention, treatment, and rehabilitation [4].

This paper is concerned with application of behavior-science knowledge in prevention; by understanding barriers to health behavior change among community patients to prevent development of diabetes and use knowledge gained as a guide for designing health promotion programs.

The process of behavior change is controlled by many factors, according to research paper by Robinson, et al. [5] examining facilitators and barriers to the heal-

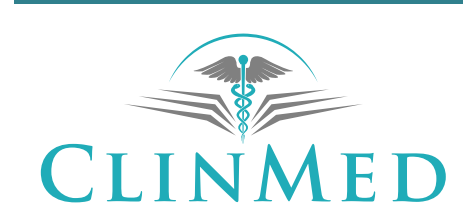

INTERNATIONAL LIBRARY 
th promotion practice:

"From an interpersonal health behavior perspective, social cognitive and/or learning theory is informative based on its assertion that behavior is a product of the interaction between individual and environmental factors; and therefore, behavior change requires supportive beliefs, training and/or skills, incentives and/or reinforcements, and social and physical environments" [5].

While the government is spending a lot of resources aiming to reduce the burden of diabetes and other non-communicable diseases (NCDs); diabetes still representing an endemic in the area and the number of cases is still high. In 2015, one million people were reported as having diabetes in the UAE [6]. The population compliance and engaging on the wide scale programs and services remain a challenge. Healthcare providers' role especially in primary care needs to expand and cover techniques like behavior change to influence individuals' decision. On the other hand, there is little research efforts done on this topic to address the problem of poor compliance to health promotion and disease prevention programs. There are studies done in the UAE to explore barriers to certain health behaviors in specific disease population. A research study done by Al-Kaabi, Al-Maskari, et al. [7] on diabetic patients to explore barriers to activities among type 2 diabetic patient found that, cultural issues, especially pertaining to women, represents main barrier to physical activity. Other barriers include the shape of traditional clothes, for both genders, that hide the excess weight and reduce the motivation to physical activity [7].

Another study that was conducted on seven Arab countries (including UAE) to highlight perceived barriers to healthy eating and physical activity among adolescences [8]. The study found number of barriers to be prominent among adolescences, barriers to healthy eating were lack of information on healthy eating, lack of motivation to eat healthy diet, and not having time to prepare or eat healthy food [8]. Lack of time was a common barrier for both healthy eating and physical activity among the seven countries.

Barriers to behavior change can be contributed to many factors. It could be -but not limited to- social, psychological, environmental, and financial. A study done on Canada on patients with diabetes to address financial barriers to care found that the main aspects which represent financial barriers were medications, diabetes supplies, and healthy food [9]. Exploring these barriers allowed the patients to seek healthcare providers aid in overcoming these barriers; demanding more thoughtful care and effective strategies to help reducing the financial barriers.

Management of diabetes type 2 revolves around many techniques, currently the most accepted method is self-management. Strategies like lifestyle modifica- tions including diet and physical activity are the main focus of self-management. In order to achieve these goals, it is not enough to educate the patients only but also to monitor their progress and understand what holds them back from meeting their health goals.

Some studies deeply explored barriers to health behavior change and identified what initiates a health behavior and what sustains it. A study by $G$ Volpp and Mohta founds that improved access to preventive care is the best strategy for initiating a health behavior whereas in-person social support is the best for sustaining the healthy behavior [10]. The study involves 775 participants in patient engagement survey done to explore what strategies initiate behavior change and what sustain the change. For long term effects and useful behavior change, the best strategy is in-person social support.

\section{Method, Sample and Data Collection}

\section{Method}

This is a qualitative survey study. A bilingual online survey (Arabic and English) has been distributed to community patients who have type 2 diabetes (Appendix 1 ). Through Ministry of Health and Prevention (MOHAP) media and communication center, the survey was sent to all MOHAP users through email. The survey was also distributed along with the electronic consent (Appendix 2 ) in health centers under MOHAP which has NCD cliniCS, diabetes clinics, and chronic disease community patients. This research has undergone Institutional Review Board IRB process from two committees: MOHAP research ethics committee in UAE and IRB in Arizona State University ASU, USA. MOHAP research ethics committee had approved the study protocol to be conducted (Reference no.: MOHAP/DB-REC/MJJ/No.20/2019).

The study was exemption granted from IRB in Arizona State University (IRB ID: STUDY00010026). The IRB determined that the protocol is considered exempt pursuant to Federal Regulations 45CFR46 (2) Tests, surveys, interviews, or observation on 4/23/2019.

\section{Sample}

The population of this study is community adults' patients with types 2 diabetes in the UAE. The ideal sample size was calculated considering population size of 1 million (based on IDF report last report in 2017 [2]). Confidence Level of $90 \%$ and margin of error $7.4 \%$ were used. The minimum acceptable sample size is 100 . Convenient sampling method was followed for collecting data for this research. A total number of 151 responses were recorded, but only 123 samples provided completed questionnaires. The uncompleted surveys were destroyed and not used in the study. 63 responses were collected online, while the other 60 responses were collected from health centers during patients' regular visits. In the case of 60 responses which were collected in 
health centers, the process of data collection was handled by the nursing team in the health care center. Any patient with type 2 diabetes who visited their community health center in the period from $26^{\text {th }}$ of April 2019 till $10^{\text {th }}$ of May 2019 was asked to participate in the survey. The sample gender distribution of the 123 participants is: 27 males and 96 females. The age group of the sample varies from 18 years to 74 years with $54.8 \%$ of the participants in the age group of 34 to 54 years (Table $1)$. The education level of the sample represents large group of educated patients, the majority has a bachelor's degree as their highest education (Figure 1). The participants were provided with an electronic consent containing brief description of the study and its importance in planning health programs and strategies for the management of DM type 2 .

The inclusion criteria are: 1) Being diagnosed with type 2 diabetes, 2) Above 18-years-old, 3) Living in the UAE, and 4) Not hospitalized during the time of taking the survey. The exclusion criteria are not meeting the inclusion criteria.

\section{Questionnaire}

The survey questions were created to explore the

Table 1: Age and gender distribution for the study.

\begin{tabular}{|l|l|l|}
\hline & Male & Female \\
\hline 18 to 34 y & 2 & 35 \\
\hline 34 to 54 y & 15 & 52 \\
\hline 55 to 74 y & 10 & 9 \\
\hline Total & 27 & 96 \\
\hline Percentages & $21.90 \%$ & $78.10 \%$ \\
\hline Grand Total & 123 & \\
\hline
\end{tabular}

real barriers to health behavior change by obtaining the point of view of patients with DM. In this study, barriers refer to any obstacles of any nature that prevent people with type 2 diabetes from changing their health behavior to a healthier one as instructed by their health providers. The questions contain inquiry about patients' perception about barriers to behavior change and effective ways to motivate behavior change. 10 questions about participant's demographics, history of disease diagnosis, barriers to behavior change, and motivators to behavior change from their personal experience are the main topics in the survey (Appendix 1). The last question focuses on actual behavior change experience in which participants succeed in changing their behavior permanently and what was the main motivator to change.

\section{Data analysis}

The raw data was collected and recorded by Qualtrics software for online surveys. After completion of data collection, the data was imported to Microsoft Excel to be analyzed. The analysis includes summary statistics for participants demographics and figures and graphs with the percentages for other survey questions.

\section{Results}

The answers to survey questions were analyzed and displayed using bars and graphs. The population characteristics are summarized on Table 1 and Figure 1. The participants' duration of DM diagnosis is shown on (Figure 2). The duration of being diagnosed with DM differs between the participants, $28.2 \%$ of the participants were newly diagnosed with diabetes (less than a year). A question about attempts to change behavior after being diagnosed with DM showed that: $69.3 \%$ of par-

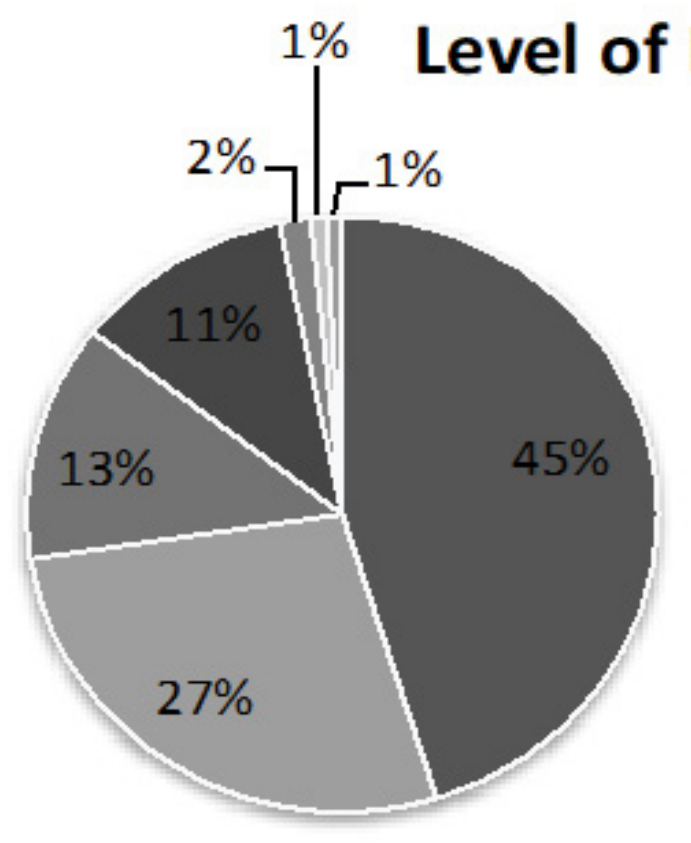

Bachelor degree

Dip loma \H igher dipl oma

Second ary school

Masters degree

Doc tor al degree

Preparatory School

Primary School

Figure 1: Level of education. 


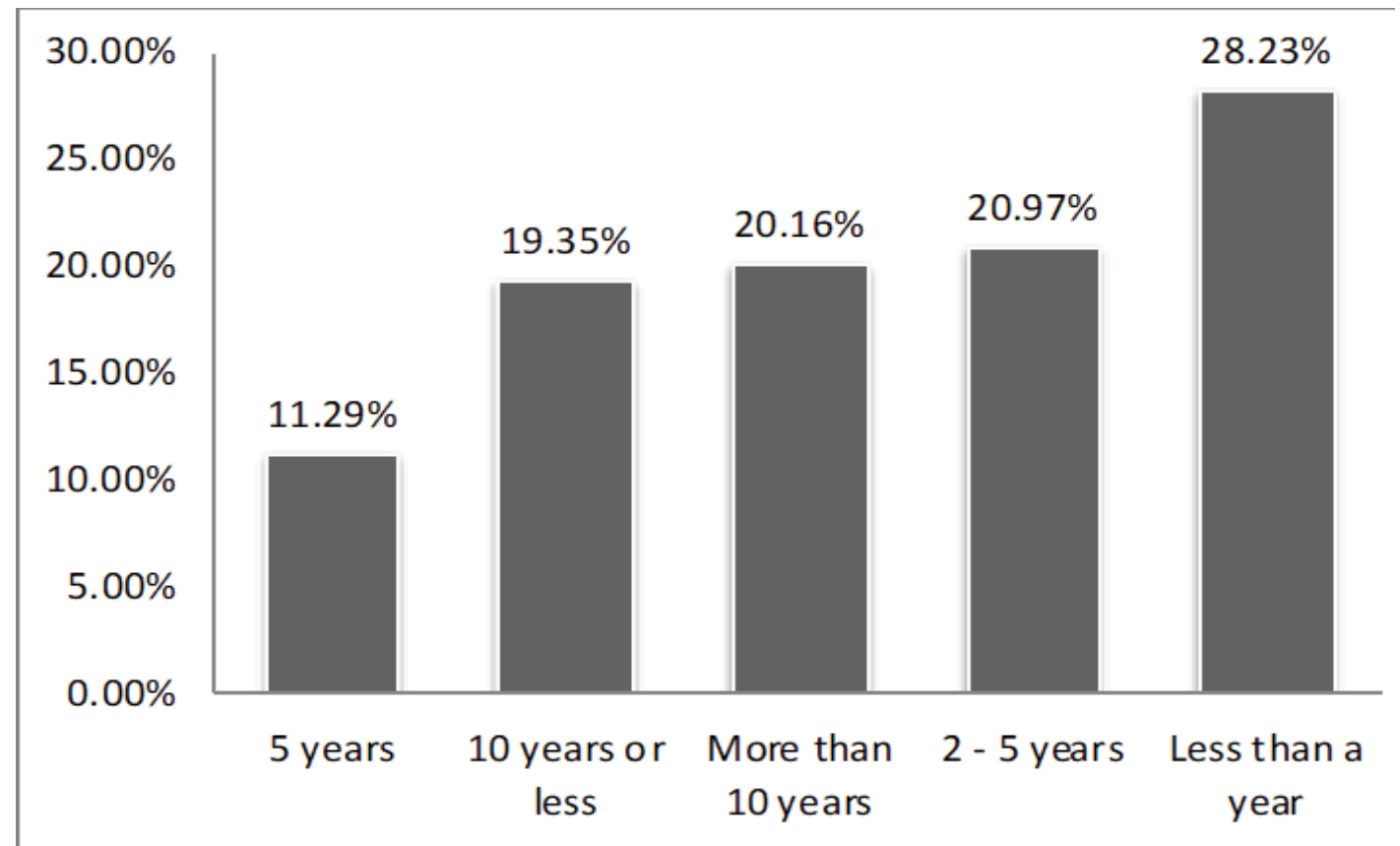

Figure 2: Duration of being diagnosed with DM type 2.

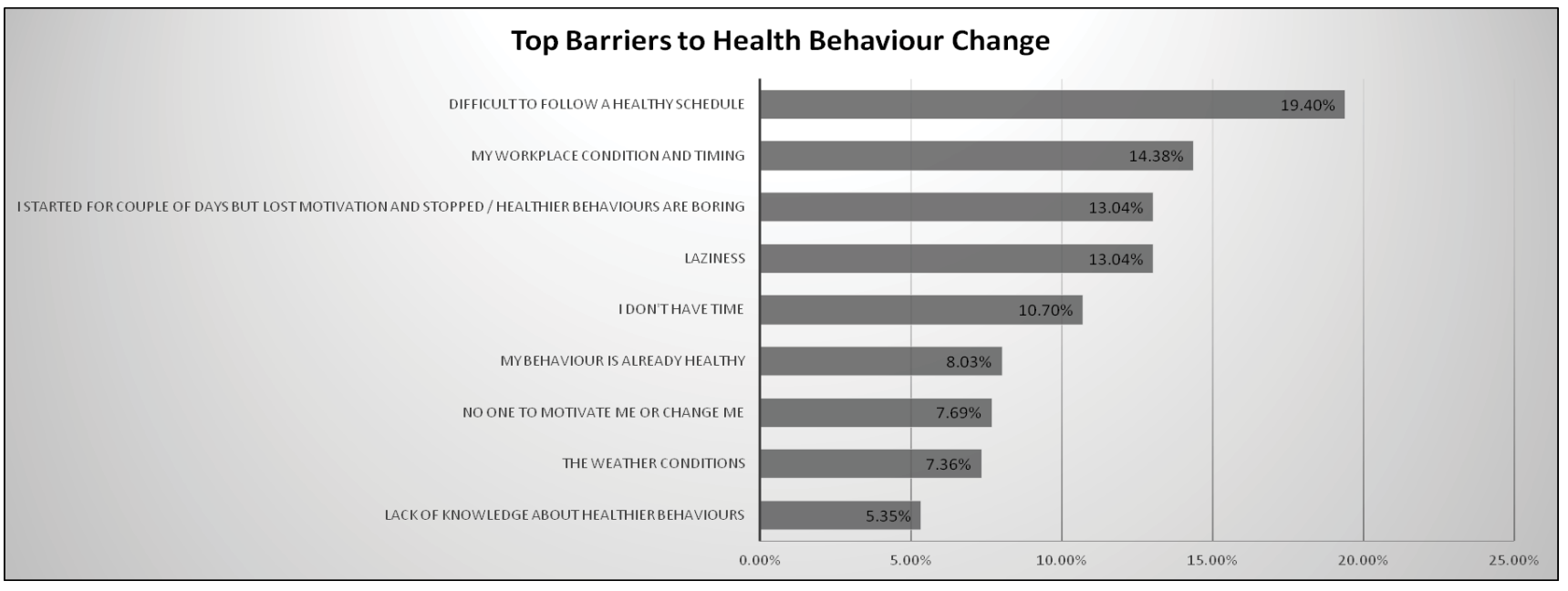

Figure 3: Percentage of top barriers to health behavior change rated by the participants.

ticipants started changing their behavior to a healthier one, where $22.7 \%$ of the participants denied changing their behavior after being diagnosed and $8 \%$ can't remember.

The core question in the survey is about ratting the top barriers to health behavior change. Participants replied by ratting the top four barriers between nine commonly reported barriers and added other barriers that were not listed in the choices (Figure 3).

The most rated barrier was the answer: "Difficult to follow a healthy schedule" $19.4 \%$, on the other hand the least rated barrier was the answer: "Lack of knowledge about healthier behaviors" 5.3\%. Other barriers like: Workplace condition, time, laziness, and loss of motivation were rated similarly on a range from $14 \%$ to $10 \%$. Being already healthy, lack of support, and weather condition were rated less than others on a range from $8 \%$ to $7 \%$.

Other barriers that were reported by the participants include: Spouse lack of knowledge and temptation of unhealthy food.

After responding to what holds them back from changing their behavior participants reported what are the best ways to motivate behavior change (Figure 4). The best way was the answer: "Doctor's advice during regular visit". $26.3 \%$ of the participants believed that doctor's advice to them during their checkups is the best way to motivate them to follow healthy behaviors. The second way is the answer: "Joining support group". $18.4 \%$ reported that the support group is essential in motivating behavior change. Motivators like receiving reminders on smartphones, weekly or monthly follow 


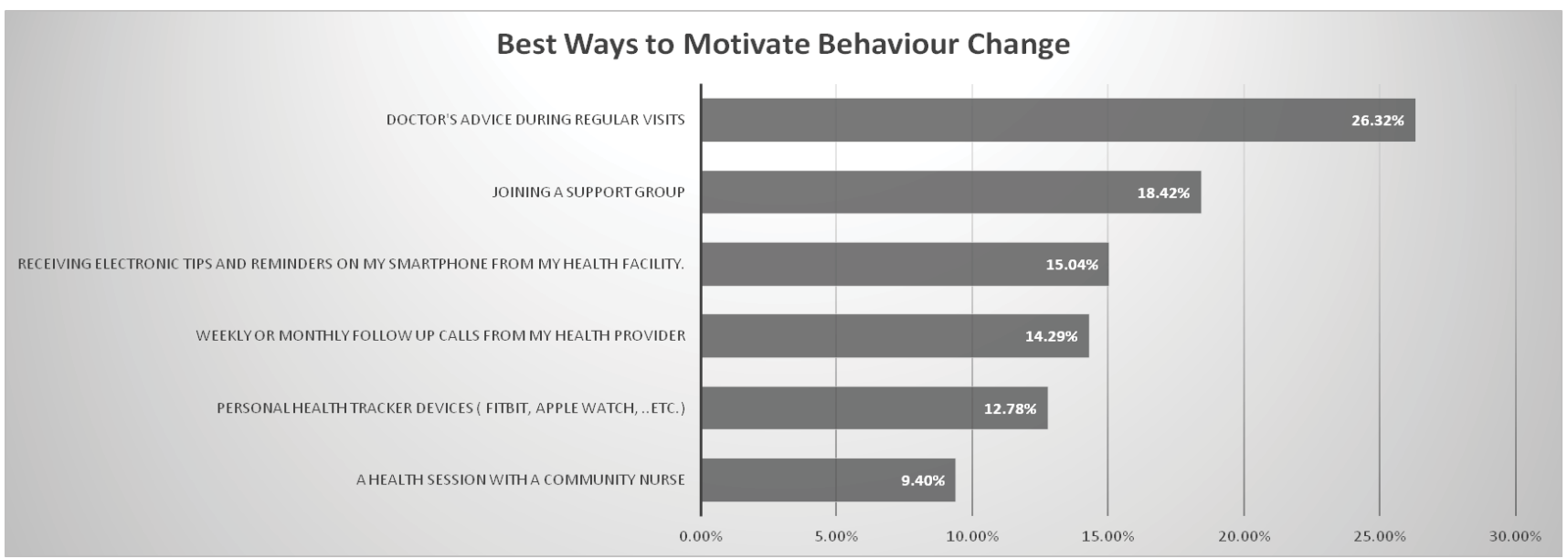

Figure 4: Percentages of effective ways in motivating behaviour change.

up calls from health facility, and personal health trackers were similar in percentages by $15.4 \%, 14.3 \%$, and $12.8 \%$ respectively. The least way to motivate was the answer: "Health session with a community nurse" by $9.4 \%$.

Other motivators that were reported by the participants are: Self-education through the internet, body shape, family support, to live longer and fear of diabetes complications.

The last question in the survey was about participants' experience in permanently changing their behavior. $72.6 \%$ of the participants actually succeed in changing their behavior permanently, where $23.4 \%$ replied that they never succeed in changing their behavior. Motivators for successfully changing the behavior were: Friends support, the presence of nutritionist in the workplace, self-determination, and childbirth.

More than $50 \%$ of the participants reported that their family represent the main motivator. The fear of leaving their children and family without support if they didn't take care of themselves, gave them a reason to change.

\section{Discussion}

This study finds that there are a lot of barriers that stands in the way of patients with diabetes. The sample contains more females than males. The level of education reflects more educated individuals which are attributed to the segment of the sample that received the online survey through MOHAP email. The duration of DM diagnosis differs between the participants, the majority were newly diagnosed and diagnosed within 10 years or less.

This mixture gives the perception of both patients who adapted to the disease management and patients who are newly adapting to DM management. Being diagnosed with DM was the start of behavior change to $69.3 \%$ of the participants. The concern of DM complications and comorbidities caused immediate change of behavior.
Barriers to behavior change ratting by the participants reflect important points. The difficulty to follow a healthy schedule was the main barrier in this study. Healthy schedule which includes lifestyle modifications and healthy diets hard to follow. Patients with diabetes receive tips and instructions from their doctor, nurse, and dietician regarding their diet. If following these instructions represents a barrier; health professionals should focus their efforts in strategies to make healthy schedule easier to follow. The active involvement of dieticians and nutrition specialists is important for overriding this barrier. On the other hand, lack of knowledge about healthier behaviors is at the bottom of the list of barriers which draws our attention to important point; patients with diabetes are well informed and have all the needed knowledge about their disease and how to manage it. This finding is consistent with other studies; a study done by Bastin, et al. [11] on barriers to change behavior on obese patients reported that:

"The vast majority of participants, regardless of the severity of obesity, know they should do and also want to do something to improve their health, but faced a lack of willpower. Thus, the most important thing to consider during an obesity intervention is the lack of motivation to modify health behaviours ....."

The resources and efforts spent on health education activities should be shifted toward healthy habits implementation and personalized methods to follow healthy schedule. The nutritionists can focus on understanding what each patient needs and tailor the healthy recommendation to their habits.

This study does not test the association between barriers and gender, level of education, and age. However, having almost half the sample $(45 \%)$ from bachelor's degree holders indicate that education level may have an effect on reducing the impact of barriers like lack of knowledge (the least reported barrier).

The second top barrier is the workplace condition and timing. Workplace conditions refer to how conve- 
nient is the place a patient with diabetes working in to facilitate maintaining health behavior. Long hours, short breaks, and lack of healthy food on the workplace are representing huge challenge for patients with diabetes. To override this barrier, employers should include the healthy habits principles in their agendas like scheduling exercise time during the working hours, offering healthy snacks, and provide nutrition counsellor if applicable.

Another way is the presence of nutritionist in the workplace to follow up and measure the progress of employees' health habits, which was reported to be one of the motivators to behavior change.

Other barriers like laziness, lack of time and loss of motivation are personal factors that can be controlled by the patient and his healthcare provider. These points should be assessed in each patient in order to be addressed if they are representing a barrier. Some participants reported that spouse lack of knowledge is one of the barriers to behavior change. Plans can be done to provide training and health education to family members.

Utilization of principles of behavior change theories and putting it into practice can be very effective in overriding barriers. The theory of planned behavior was used by nurse practitioners to develop a framework that tailored strategies for diabetes self-management [12]. They used the central concept of the theory which is "intention" as the best predictor for behavioral change and start by assessing it very early in the treatment [12]. The paper highlighted the need for high-quality care, which include strategies to address psychological barriers to adhering to diabetes self-management programs [12].

When analyzing the best ways to motivate behavior change, doctors' advice during regular visits was the best motivator. Patients tend to value the doctor's advice and pay more attention to it. This finding is consistent with findings of other studies which linked patient's adherence to medical care and their trust in the medical profession [13]. Doctors must maintain good practice in assessing and addressing barriers to health behavior change and develop plans with the help of patients. The second-best way is joining a support group. The role of support group in motivating behavior change and override barriers is tremendous. Support group concept needs to be well established and utilized here in the UAE. Volunteers association and health facilities can establish support groups and recommend it to promote behavior change.

The other motivators can be categorized under the use of technology in changing the behavior: Sending reminders and tips to patients' smartphones by the health facility, weekly and monthly follow up calls by the healthcare provider, and personal health trackers. These methods were reported by participates to be the effective in motivating behavior change. Weekly or mon- thly follow up calls are proved to be effective in sustaining behavior change by other studies [11]. This method represents in-person social support.

The least method for motivating behavior change was health session with community nurse. Although the role of nurses in health education and promotion is known to be effective by many studies; this study finds that participants favor other ways of motivation than health session with a community nurse. It is recommended to fully utilize the nurse knowledge and expertise in counseling and dealing with patients who have chronic diseases including diabetes mellitus.

According to WHO recommendation; the fact that nurses are the largest group of health-care providers, they can make an important contribution to efforts to prevent, reduce and treat noncommunicable diseases (NCDs) [14].

Strategies like proper training and introduction of nurse led NCD clinics can have huge impact in changing population health behavior.

\section{Conclusion}

The outcomes of this study can aid directly in community programs design and implementation, health education campaigns, and health policies. The findings of this study help in understanding real barriers to initiating and sustaining positive health behavior. Healthcare professionals should work with patients with diabetes by assessing and addressing the top barriers which is facing this type of patients.

\section{Limitation}

The study has some limitations in terms of recruiting participants and data collection. Online surveys have the advantage of reaching out more responses but result on bias; only individuals who has access to the internet and are literate enough to answer the survey had the chance to participate. For this type of research, the best method of data collection is face-to-face interactive interview to capture more authentic responses and avoid reply bias.

For future research, it is recommended to conduct more studies on the area of behavioral sciences and its implication in the UAE. Designing interventions for health promotion program using the findings of this study is recommended. Strategies to Involve all health professionals especially dieticians in changing the behavior of patients with diabetes. More Nursing research to study utilization of nurse-led intervention in changing behavior is recommended.

It is also important to use the technology and innovative methods to motivate, initiate and sustain healthy behavior.

\section{Acknowledgment}

This research was supervised by Professor Kristen 
Will: (MHPE, PA-C Director, Executive and Continuing Education, Clinical Assistant Professor, Science of Health Care Delivery Arizona State University | College of Health Solutions) as a principal investigator. This is to extend special thanks and appreciation for her guidance and expertise which added to the quality of this research study.

This is also to extend many Thanks and appreciation to the nursing team in Sharjah primary health care centers, ministry of health and prevention for supporting and participating in the process of data collection.

\section{Statement of Ethics}

This research has undergone Institutional Review Board IRB process from two committees: MOHAP research ethics committee in UAE and IRB in Arizona State University ASU, USA. MOHAP research ethics committee had approved the study protocol to be conducted (Reference no.: MOHAP/DB-REC/MJJ/No.20/2019).

The study was exemption granted from IRB in Arizona State University (IRB ID: STUDY00010026). The IRB determined that the protocol is considered exempt pursuant to Federal Regulations 45CFR46 (2) Tests, surveys, interviews, or observation on 4/23/2019.

The participants were provided with an electronic consent containing brief description of the study and its importance in planning health programs and strategies for the management of DM type 2 prior to answering the survey.

\section{Disclosure Statement}

The author has no conflict of interest to declare.

\section{Funding Sources}

No funding resources or sponsors.

\section{References}

1. Jelinek HF, Osman WM, Khandoker AH, Khalaf K, Lee S, et al. (2017) Clinical profiles, comorbidities and complications of type 2 diabetes mellitus in patients from United Arab Emirates. BMJ Open Diabetes Res Care 5: e000427.
2. Sulaiman $\mathrm{N}$, Elbadawi S, Hussein A, Abusnana S, Madani A, et al. (2017) Prevalence of overweight and obesity in United Arab Emirates Expatriates: The UAE national Diabetes and Lifestyle Study. Diabetol Metab Syndr 9: 88.

3. International Diabetes Federation (2017) Recommendations for managing type 2 diabetes in primary care.

4. McCracken, History, context, and new developments in behavioral medicine. Centre for Pain Services, Royal National Hospital for Rheumatic Diseases and Centre for Pain Research, University of Bath, Bath, United Kingdom.

5. Ajzen I (2019) Theory of planned behavior website.

6. Robinson KL, Driedger MS, Elliott SJ, John E (2006) Understanding facilitators of and barriers to health promotion practice. Health Promot Pract 7: 467-476.

7. Al-Kaabi J, Al-Maskari F, Saadi H, Afandi B, Parkar H, et al. (2009) Physical activity and reported barriers to activity among type 2 diabetic patients in the United Arab Emirates, the review of diabetic studies. Rev Diabet Stud 6: 271-278.

8. Abdulrahman $O$ Musaiger, Mariam Al-Mannai, Reema Tayyem, Osama Al-Lalla, Essa Y A Ali, et al. (2013) Perceived barriers to healthy eating and physical activity among adolescents in seven Arab Countries: A cross-cultural study. The Scientific World Journal 2013: 232164.

9. David JT Campbell, Braden J Manns, Brenda R Hemmelgarn, Claudia Sanmartin, Alun Edwards, et al. (2017) Understanding financial barriers to care in patients with diabetes. Diabetes Educ 43: 78-86.

10. G Volpp K, Mohta NS (2018) Patient engagement survey: What creates behavior change may not sustain it. NEJM Group.

11. Bastin A, Romain AJ, Marleau J, Baillot A (2019) Health behaviours, intentions and barriers to change among obesity classes I,II and III. Clin Obes 9: e12287.

12. Lee LT, Bowen PG, Mosley Mk, Turner CC (2017) Theory of planned behavior: Social support and diabetes self-management. The Journal for Nurse Practitioners.

13. Trachtenberg F, Dugan E, Hall MA (2005) How patients' trust relates to their involvement in medical care. J Fam Pract 54: 344-352.

14. WHO (2013) Enhancing nursing and midwifery capacity to contribute to the prevention, treatment and management of non-communicable diseases. 